\title{
MEMÓRIA DE TRABALHO: QUE IMPORTÂNCIA TEM NA APRENDIZAGEM E NO PROCESSAMENTO DA LINGUAGEM?
}

\author{
WORKING MEMORY: WHAT RELEVANCE DOES IT HAVE IN \\ LEARNING PROCESS AND IN LANGUAGE PROCESSING?
}

Submetido em: 01-04-2012

Publicado em:23-02- 2013

Lidiomar José Mascarello ${ }^{1}$

lidiomarjose@gmail.com

\begin{abstract}
Resumo: Trata-se de uma revisão sistemática de literatura e publicações acerca do tema memória de trabalho. As pesquisas, entre elas a de George Miller (1956) e Paul CarrilloMora (2010) sobre sistemas de memória, identificaram que a memória de trabalho ajuda a lembrar informações visuais e espaciais, além de coordenar as atividades cognitivas e planejar estratégias. Inicialmente examinam-se alguns fatos importantes na história da pesquisa em memória de trabalho e em seguida faz-se uma analise de trabalhos publicados no período de 2001 a 2011 e disponíveis na Scientific Eletronic Library Online (Biblioteca Científica Eletrônica em Linha). Encontramos diferentes pesquisas voltadas para diferentes aspectos da memória de trabalho. Em relação à linguagem, parece que há um maior número de trabalhos destinados ao estudo de aspectos fonológicos. Há ainda pesquisas que tratam da relação da memória de trabalho e os aspectos gerais da inteligência. Outros estão voltados para o déficit de aprendizagem e outros prejuízos causados por problemas de memória. Ainda não se tem respostas suficientes, ou seja, não está consolidada a relação entre déficit de aprendizagem e a capacidade de manutenção da memória de trabalho, mas já é consenso entre os pesquisadores que a quantidade de informação linguística que podemos manter na memória de trabalho é influenciada pelo tempo de pronúncia e pela similaridade semântica dos termos. Os estudos analisados apontam para as mesmas conclusões.
\end{abstract}

Palavras-chave: Sistema de Memória. Memória de Trabalho.

\begin{abstract}
This work consists of a systematic review of the literature on working memory. Researches, including the ones developed by George Miller (1956) and Paul Carrillo-Mora (2010) have shown that working memory is involved in remembering visual and spatial information, as well as in cognitive activities and in planning strategies. In the present article, we first examine some important facts in the history of research about working memory. After that, we analyze works published from 2001 to 2011 and available at the "Scientific Electronic Library Online". During this process, we found studies about different aspects of working memory, but those related to language are usually focused on phonological aspects. There are some works about the relation between working memory and general aspects of intelligence, while others are about difficulties in learning and problems related to memory. We still do not have conclusive
\end{abstract}

\footnotetext{
${ }^{1}$ lidiomarjose@gmail.com. Pós-Graduação em Linguística, UFSC.
} 
answers, in other words, the relation between problems in learning and working memory capacity is not widely accepted; however, there is a consensus that the amount of linguistic information we can keep in our working memory is influenced by spelling time and semantic similarity of the terms used. The studies analyzed here point to the same conclusions.

Keywords: Memory system. Working Memory. Relations.

\section{INTRODUÇÃO}

Antes de falarmos diretamente do conceito de memória de trabalho é necessário explorar, ainda que brevemente, a história do conceito destes termos.

$\mathrm{Na}$ literatura voltada para esta área um nome que não podemos esquecer é o de George Miller, que escreveu um artigo intitulado "The magical Number Seven, Plus or Minus Two: Some Limits on Our Capacity for Processing Information" em 1956 (apud MATLIN, 2004, p.53). O pesquisador propõe que podemos reter somente um número limitado de itens na memória de curto prazo, como era denominada a memória de curta duração. Ele sugeriu que as pessoas podem lembrar cerca de sete itens com uma pequena variação, acrescentando mais ou menos dois itens. George Miller (1956) propôs a noção de um agrupamento, ou seja, uma unidade cognitiva composta de poucos componentes que representava um padrão perceptual frequente. Este agrupamento pode ser representado por um número ou uma letra, sendo que os números e as letras podem ser agrupados em unidade maiores como código de área (CEP), número de telefone ou palavras, no caso das letras.

Uma pesquisa clássica sobre a memória de curto prazo foi a pesquisa de Brown/Peterson \& Peterson (1958 apud MATLIN, 2004, p. 54). Estes pesquisadores demonstraram através de experimentos que informações retidas por menos de um minuto na memória de trabalho tendem a ser com frequência esquecidas. A partir destas descobertas Brown/Peterson \& Peterson criaram uma técnica em 1959 que leva o próprio nome deles. Eles solicitavam nessa técnica, por exemplo, que as pessoas estudassem uma lista composta por três letras, também chamada de trigramas, seguidas por um número de três dígitos. Os participantes deveriam contar de trás para diante, a partir do número escolhido, por um período curto de tempo, essa atividade impedia que fossem repetidas silenciosamente as séries de estímulos durante o intervalo de retenção.

Outro estudo relevante sobre memória de curto prazo é a de Rundus (1971, apud MATLIN, 2004, p. 55), que trata do efeito de posição serial, (a expressão 'efeito de 
posição serial' é usada para referenciar a relação em forma de U que se dá entre a posição de uma palavra em uma lista e sua possibilidade de ser recortada), e do efeito de recenticidade (está relacionado ao efeito serial, a curva em forma de U expõe um efeito de recenticidade, isto é, uma melhor recordação dos itens que se encontram no final da lista).

Estudos de Atkinson e Shiffrin (1968, apud MATLIN, 2004, p. 56), também são importantes por tratarem de conceito de memória de curto prazo. Os autores defendiam que as lembranças na memória de curto prazo são frágeis e que poderiam perder-se em cerca de trinta segundos, caso não fossem repetidas. Mas mais que isso, Atkinson e Shiffrin (1968) desenvolveram um modelo de processamento de informações, em outras palavras, um modelo de memória. Este modelo focava o papel da memória de curto prazo na aprendizagem e na retenção da informação.

Durante as décadas de 1950 e 1960, a maior parte das pesquisas e das teorias sobre a memória de curto prazo tinha sido produzida nos Estados Unidos. Entretanto, na década de 1970, um novo movimento sobre estudos da memória se desencadeia e se desenvolve no Reino Unido. Dentre os pesquisadores britânicos destacam-se Alan Baddeley e colaboradores. Em 1974 Baddeley e Hitch (apud MATLIN, 2004, p. 56) propõe um novo termo para memória de curto prazo, definindo-a como memória de trabalho, o que hoje é aceito pelos pesquisadores (embora seja simplista afirmar isso, pois não se trata apenas da substituição de termos, uma vez que a memória de trabalho é considerada mais flexível e com características diferentes da memória de curto prazo). Além disso, Baddeley e Hitch (1974) propõem um novo modelo e uma nova perspectiva para o estudo da memória. O termo memória de trabalho é tido atualmente como termo padrão. Segundo Baddeley e Hitch (1974), a memória de trabalho possui três componentes distintos, cada um com capacidade independente:

1 - o circuito (loop) fonológico, que armazena um número limitado de sons por um período curto;

2 - o bloco de esboço (sketch pad) visuoespacial, que armazena informações visuais e espaciais;

3 - o executivo central, que integra as informações oriundas dos outros dois componentes, bem como a memória de longo prazo. (MATLIN, 2004, p. 56)

Ao tratar de aspectos mais específicos da linguagem, desde as pesquisas da década de 1950, especialmente as pesquisas de Miller (1956, apud MATLIN, 2004, p. 57) sugerem que todos os agrupamentos feitos e armazenados na memória são de certa 
forma comparáveis, e, uma das hipóteses desde então defendida é que a capacidade de memória de trabalho é influenciada pelo tempo de pronúncia e pela similaridade semântica dos termos.

As pesquisas de Baddeley, desde a década de 1970, aperfeiçoaram e aprofundaram os estudos sobre os processos cognitivos. Baddeley defende que a memória de trabalho apresenta diversos componentes que podem agir de maneira parcialmente independente uns dos outros. Essa nova perspectiva sobre memória de trabalho abre portas para muitas pesquisas na área. Os teóricos da memória concordam hoje em que a memória de trabalho não pode ser unitária. Com o intuito de buscar evidencias sobre o que está sendo pesquisado e de que forma estão sendo realizadas as pesquisas sobre memória de trabalho e a aprendizagem da linguagem na America Latina e no Caribe, para tal, nos propomos a realizar uma 'varredura' em busca de informações. Provavelmente não identificaremos todas as pesquisas realizadas, o que também não é a pretensão.

\section{MATERIAIS E MÉTODOS}

Com o objetivo de averiguar quais são as linhas de pesquisas e os trabalhos publicados sobre memória de trabalho realizados na America Latina e Caribe utilizou-se como base trabalhos/artigos publicados na base de dados da SciELO. Foram selecionados e analisados 25 (vinte e cinco) trabalhos publicados no período 2001 a 2011. Foram utilizados dois critérios para a seleção: um dos critérios de seleção foi apresentar no título ou no resumo o termo memória de trabalho articulado a algum fator de aprendizagem e/ou aspectos da linguagem. Outro critério foi o local de publicação, restrito a America Latina e Caribe. Utilizou-se também dois livros, um referente à Psicologia Cognitiva e outro referente a Fundamentos da neurociência e do comportamento como apoio teórico para estudo do conceito de memória de trabalho.

Foi utilizado, principalmente, o método de pesquisa online com o auxílio do programa Mozilla Firefox.

\subsection{Metodologia}

Em primeiro lugar foi realizada a seleção e o recorte do tema da pesquisa a partir das aulas da disciplina Linguagem e Cérebro. Segundo, revisão de referência

Work. pap. linguíst., 13(3): 87-105, Florianópolis, out.dez,2012 
bibliográfica, busca em livros e artigos publicados em periódicos online no período de 2001 até 2011. A base da pesquisa se deu a partir do site da SciELO, que é uma base de dados especializada em publicação e apoio a pesquisa científica, trabalhando de forma cooperativa para assegurar a visibilidade da pesquisa nos países em desenvolvimento, particularmente da América Latina e Caribe.

\subsection{Análise dos dados}

Classificando os artigos pesquisados a partir de seus objetos de análise, formamos três grupos. O primeiro grupo: Memória de trabalho e fator $\mathrm{g}$ (fator geral de inteligência) foram localizados 11 estudos: Colom, Mendoza (2001); Colom, Mendoza (2006); Crespo Allende, Alvarado Barra, (2010); Eckschmidt, et al. (2007); Lopes et al. (2005); Mansilla-Olivares, (2005); Miranda et al (2006); Primi (2002); Tessmann Bandeira (2008); Vasconcelos, Albuquerque (2011); Vaz, Cordeiro, Macedo (2010). O segundo grupo: Prejuízos de memória (de diversas formas), foram localizados 4 estudos: Belarmino et. al (2009); Fukuda et. al (2010); Lukasova et. al (2009); Messina e Tiedemann (2009). O terceiro grupo: trabalhos que visam analisar aspectos mais específicos e diretamente associados a capacidades linguísticas, foram localizados 10 estudos: Crespo Allende, Alvarado Barra (2010); Eckschmidt et al. (2007); Gendri et al. (2007); Gombert (1992); Lopes et al. (2005); Rodrigues Lopes (2009); Silveira Finardi (2011); Skehan, (1998); Tessmann Bandeira (2008); Vaz et. al (2010). A seguir destacaremos alguns aspectos de cada grupo.

2.2.1 Memória de trabalho e fator geral de inteligência

O primeiro trabalho publicado sobre Memória de trabalho e fator $\mathrm{g}$, no período selecionado, foi de uma equipe de pesquisadores da Universidade Federal de Minas Gerais (UFMG), Colom e Mendoza (2001). Os autores afirmavam em suas pesquisas que há relação entre o fator g e a complexidade cognitiva associada à capacidade de processamento. As bases teóricas por eles utilizadas foram da psicologia experimental, que tem estudado extensivamente as características da memória, e a psicologia correlacional que se dedica a análise das principais propriedades da inteligência. A aproximação entre os enfoques foi baseada na análise das relações entre um conceito nuclear da memória - a memória de trabalho - e o principal ingrediente do conceito psicométrico de inteligência - o fator $g$. Segundo Colom e Mendoza (2001), as

Work. pap. linguíst., 13(3): 87-105, Florianópolis, out.dez,2012 
evidências disponíveis sugerem que as diferenças individuais em $g$ poderiam ser explicadas pelos conceitos cognitivos de capacidade e de velocidade associados à memória de trabalho. Essas evidências sugerem novos modos de melhorar a inteligência e, portanto, os correlatos associados a ela através do incremento da capacidade do sistema para processar informação de maneira eficiente. Em 2006 os mesmos pesquisadores, Colom e Mendoza (2006), ampliaram suas pesquisas sobre o tema e apresentam a discussão das evidências empíricas derivadas da análise das relações entre o processamento humano de informações e da psicologia das diferenças individuais, ou seja, entre a memória de trabalho e o fator geral de inteligência $(\mathrm{g})$.

Os resultados publicados por Colom e Mendoza (2006), avaliam a presença de um isomorfismo entre os construtos, embora, segundo os autores, ainda se desconheça a resposta à pergunta sobre o porquê desse isomorfismo. Segundo os autores, Colom e Mendoza (2006), algumas propostas favorecem uma explicação baseada em construtos tais como o controle da atenção, outras se inclinam pelo papel da capacidade para armazenar transitoriamente a informação e, em menor grau, da velocidade de processamento. O estudo de Colom e Mendoza (2006) descreve as principais discussões acadêmicas e apresentam os resultados obtidos em recentes investigações conduzidas por eles. Os pesquisadores concluem que os componentes de armazenamento e velocidade de processamento podem constituir a fonte de explicação para a relação entre o construto de memória de trabalho e o fator $g$.

Ainda em relação aos estudos da memória e o fator geral de inteligência, Miranda et al (2006) realizaram um estudo acerca da importância da memória de trabalho e a gestão do conhecimento segundo a visão histórica e a análise contextual. Afirmam que os estudos da neurociência têm contribuído significativamente para a construção e o entendimento dos processos cognitivos, bem como para o aperfeiçoamento do conhecimento e da tomada de decisão. Os autores afirmam também que a memória está relacionada de forma direta aos processos de aprendizagem e este processo de armazenamento de conhecimentos nos permite a orientação no tempo e no espaço.

Associado ao fator geral de inteligência estudos de Primi (2002), dedicados à revisão do conceito de inteligência fluida, apresentam resultados das relações estabelecidas a partir de abordagens psicométrica, cognitiva e neurociência cognitiva da associação entre a inteligência fluida e seis funções componentes da memória de trabalho: 
(a) manutenção do nível de ativação das representações mentais, (b) coordenação de atividades mentais simultâneas, (c) monitoramento e supervisão das atividades mentais, (d) controle da atenção e atenção seletiva, (e) ativação de informações da memória de longo prazo e (f) redirecionamento de rotas ou flexibilidade adaptativa. (PRIMI, 2002, p. 16)

Primi conclui que as variâncias comportamentais demonstradas nos estudos psicométricos apresentam quatro fatores do modelo $\mathrm{CHC}$ : inteligência fluida $(G f)$, memória de curto prazo (Gsm), armazenamento e recuperação da memória de longo prazo $(G l r)$ e processamento visual $(G v)$, sendo que estes fatores estão ligados às funções do executivo central. Outra conclusão apontada por Primi (2002) é que há um amplo conjunto de evidências que apóiam a validade do construto de tarefas de raciocínio analógico para medir funções do executivo central. Neste sentido o autor afirma que novos instrumentos com uma definição mais clara sobre o que avaliam poderão ser criados por intermédio da aplicação do conhecimento acumulado pela psicologia cognitiva e pela neurociência.

\subsubsection{Prejuízos na memória}

Outro grupo de trabalhos selecionados para a realização do levantamento de informações é constituído de estudos que se dedicam a descobrir aspectos ligados a prejuízos sofridos pelos sujeitos, como por exemplo, déficit de atenção, esquizofrenia, síndrome epiléptica, dislexia, mal de Parkinson, tais estudos estão mais associados a grupos de estudos da psicologia cognitiva e centros de saúde.

Diferentes grupos de pesquisadores e estudiosos dedicam-se a pesquisa na tentativa de descobrir evidências na relação entre a memória e o déficit de atenção e hiperatividade. Messina e Tiedemann (2009), investigaram as habilidades cognitivas da memória de trabalho de crianças e adolescentes com "Transtorno do Déficit de Atenção e Hiperatividade" (TDAH), aplicando o teste TIHC - Teste Infantil de Habilidades Cognitivas informatizado. Este teste avalia principalmente cinco habilidades cognitivas: Raciocínio Indutivas (RI), Memória de Armazenamento Auditiva (MAA) e Visual e Memória de Trabalho Auditiva (MTA) e Visual (MTV) a partir do modelo de CattellHorn-Carroll. Messina e Tiedemann (2009) constataram que as crianças com TDAH obtiveram bom desempenho nas provas de memória visual em detrimento as provas de memória auditiva. As correlações dos resultados do TIHC verificaram que o tempo de 
reação da prova de memória de armazenamento visual manteve uma correlação significativa com a prova de cálculo. O teste foi aplicado em 62 sujeitos sendo $32 \mathrm{com}$ TDAH do Hospital das Clínicas-SEPIA-USP e 30 sujeitos controle, estudantes do Ensino Fundamental da rede municipal de $1^{\mathrm{a}}$ à $8^{\mathrm{a}}$ séries com idades entre 7 e 15 anos, de ambos os sexos. As análises executadas indicaram a existência de diferenças em diversos aspectos relacionados à memória nos tipos peculiares de crianças com TDAH, como por exemplo, dificuldades de leitura e baixa capacidade de concentração na realização de tarefas.

Outro problema enfrentado por alguns indivíduos é a esquizofrenia. Belarmino et. al (2009) ao pesquisarem e realizarem avaliação da relação entre memória de trabalho e esquizofrenia constataram que os prejuízos são múltiplos envolvendo diversos aspectos de diferentes habilidades e funções executivas. Os principais objetivos da pesquisa eram: avaliar a memória de trabalho auditiva e visual na esquizofrenia; identificar se estas duas habilidades operam como dois sistemas separados e relacionar possíveis déficits de memória de trabalho com habilidades de funções executivas. Belarmino et. al (2009) constataram que existem poucos estudos realizados no Brasil e poucas amostras brasileiras de pacientes com esquizofrenia. Belarmino et. Al (2009) incluíram em suas pesquisas 20 indivíduos com esquizofrenia e 20 indivíduos saudáveis pareados quanto a sexo, idade e escolaridade. As habilidades avaliadas foram memória de trabalho auditiva e visual, atenção seletiva, controle inibitório, flexibilidade cognitiva e planejamento. E chegaram aos seguintes resultados:

Os pacientes demonstraram prejuízos em todas as medidas dos testes, exceto em tempo de reação de controle inibitório. Pacientes apresentaram significante correlação entre MT auditiva com medidas de atenção seletiva, controle inibitório, flexibilidade e planejamento. Memória de trabalho visual apresentou correlações com planejamento e flexibilidade. (BELARMINO et. al 2009, p. 1)

Concluíram que déficits em memória de trabalho e funções executivas estão presentes em pacientes esquizofrênicos na amostra brasileira avaliada. Alterações nas funções executivas podem levar a incapacidade de adequadas operações de memória de trabalho.

Fukuda et. al (2010) publicaram resultados de suas pesquisas sobre a caracterização da linguagem e memória de trabalho em pacientes com síndrome epiléptica mioclônica astática. A intenção das pesquisadoras era avaliar a memória de trabalho fonológica (MTF) e a linguagem verbal de pacientes com SEMA (síndrome 
epiléptica mioclônica astática). $\mathrm{O}$ experimento avaliou seis pacientes entre 8 e 18 anos em tratamento há mais de 5 anos que apresentavam bom controle das crises. Estes sujeitos eram pacientes atendidos pelo Serviço de Epilepsia do Hospital das Clínicas da Faculdade de Medicina de Ribeirão Preto da Universidade de São Paulo. As pesquisadoras utilizam como instrumento para a coleta de dados a Prova de Repetição de Palavras Sem Significado. Tal instrumento foi utilizada para avaliar a memória de trabalho fonológica, e foram coletadas amostras de linguagem, gravadas em vídeo, durante atividades espontâneas e dirigidas para estudo da linguagem verbal e pragmática. A partir da análise qualitativa os dados mostraram que todos apresentaram deficiência na MTF e, exceto um, mostraram domínio dos aspectos verbais estudados. “Os achados contribuem para estratégias de tratamento de problemas de linguagem de pacientes com SEMA, com maior enfoque na MTF” (FUKUDA et al, 2010) .

Em um estudo intitulado "discriminação fonológica e memória em crianças com dislexia e bons leitores", Lukasova et. al (2009) avaliaram a capacidade de discriminação fonológica de sílabas em crianças com dislexia do desenvolvimento comparado com indivíduos que não apresentavam essa dificuldade, denominados de bons leitores. Participaram do estudo 10 indivíduos que apresentavam dislexia e 10 indivíduos controle. Foram controlados os fatores idade, sexo, série, nível intelectual e atencional. Foram apresentados para julgamento de similaridade em provas SOM/SOM e SOM/ESCRITA 45 pares de sílabas diferentes quanto à sonorização, ponto de articulação e sílabas iguais. O intervalo entre estímulos variou de curto, médio e longo. Segundo os autores,

Os resultados mostraram diferenças entre-grupo em tarefa SOM/SOM, sendo que os disléxicos obtiveram desempenho inferior aos bons leitores em sílabas sonoras e ponto de articulação com intervalos longos. Análise dos pares de sílabas mostrou baixo acerto dos disléxicos nas sonoras (/KA/-/GA/ e /FA/-/VA/) e ponto de articulação (/ZA/-/VA/). Os resultados mostram perda mais rápida da informação fonológica na memória de trabalho em crianças disléxicas. Lukasova et. al 2009, p.2)

Mal de Parkinson, outro dos prejuízos sofridos por indivíduos também é estudado e associado a problemas de memória de trabalho. Beato et. al (2008) em suas pesquisas apresentam um estudo intitulado: "Memória de trabalho em pacientes com doença de Parkinson: características clínicas e resposta a levodopa”, afirmam que pacientes com doença de Parkinson apresentam desempenho inferior ao dos controles em tarefas de memória de trabalho. O estudo baseou-se nas tarefas 'n-back' (WM com 
variação de complexidade incluindo manipulação mental e alocação de recursos atencionais e domínio de posições espaciais, faces e letras. Cada indivíduo deveria cumprir nove tarefas), que permitem avaliar o nível de demanda executiva (três níveis de dificuldade) e o domínio da informação sendo processado (posições espaciais, faces e letras). O efeito da levodopa foi estudado pela testagem dos pacientes no estado "on" e "off" (on sob efeito dopaminérgico, off depois de uma retirada de 12 horas de medicação dopaminérgica). Os pesquisadores afirmam também que não foi observada interação entre grupos e complexidade. A terapia com levodopa mostrou efeito positivo sobre a modalidade espacial, e nenhum efeito sobre a complexidade. Na conclusão do estudo Beato et. al(2008) afirmam: "Nossos resultados sugerem que o comprometimento observado pode resultar de défict de manutenção da MT, independente do nível de processamento. A terapia com levodopa apresenta efeito positivo sobre a MT espacial”.

\subsubsection{Memória e capacidade linguística}

Um terceiro grupo selecionado foi de trabalhos que visam analisar aspectos mais específicos e diretamente associados a capacidades linguísticas, como por exemplo, o desenvolvimento de regras linguísticas, a consciência metapragmática, diferenças entre crianças bilíngues e monolíngues, consciência fonológica a gestão do conhecimento para habilidades de leitura e escrita.

Em relação à aquisição e à capacidade de desenvolvimento de regras linguísticas, Silveira e Finardi (2011) publicaram um trabalho intitulado "Capacidade de memória de trabalho na produção e aquisição de uma regra sintática na fala em L2", as autoras chamam atenção para os aspectos da memória relacionados à produção e à aquisição de uma estrutura sintática na fala de uma segunda língua. Baseadas na Teoria do Processamento da Informação que afirma: à medida que se pressupõe a existência de um sistema cognitivo de código duplo, constituído de um sistema baseado em regras e outro baseado na memória (SKEHAN, 1998), sendo que ambos os sistemas são responsáveis tanto pela análise quanto pela síntese da linguagem. A aquisição da L2 também é discutida no que diz respeito ao processamento de input para o significado e para a forma. Os resultados indicam que a capacidade da memória de trabalho está relacionada com a produção e a aquisição de uma estrutura sintática na fala da L2, mas esse relacionamento é mais forte na aquisição do que na retenção de L2. 
Crespo e Alvarado (2010) ao pesquisarem sobre a consciência metapragmática (a capacidade de um sujeito para relações entre linguagem oral e contexto da mensagem extralinguística), Gombert (1992) e a memória de trabalho de crianças com idade escolar concluíram que a consciência metapragmática não depende somente do desenvolvimento linguístico dos indivíduos, mas também de uma maturação dos sistemas cognitivos no armazenamento e no processamento da informação. A pesquisa foi realizada com oitenta e oito (88) crianças com idade entre oito (8) e nove (9) anos, ambas variáveis foram medidas: consciência metapragmática e memória de trabalho. Os pesquisadores sustentam que há uma influência importante da memória de trabalho na maturação dos sistemas envolvida no processamento cognitivo e armazenamento de informação, responsáveis pela sustentação do desenvolvimento da capacidade que os indivíduos têm para estabelecer relações entre uma mensagem linguística oral e o seu contexto extralinguístico.

Um estudo de Tessmann (2008) compara o desempenho entre crianças bilíngues e crianças monolíngues em tarefas que envolvem memória de trabalho. Objetivo principal da pesquisa era averiguar se havia diferenças de atenção e inibição. A investigadora utilizou como ferramenta para a coleta de dados o teste Simon Task em dois grupos de crianças, um monolíngue outro bilíngue. Os resultados apontam que crianças bilíngues respondem mais rapidamente as tarefas que envolvem funções executivas e de controle ligadas a memória de trabalho do às crianças monolíngues.

Gendri et al (2007) ao pesquisarem memória de trabalho, consciência fonológica e escrita, com o objetivo de verificar a relação entre a memória de trabalho, a consciência fonológica e a hipótese de escrita em alunos de pré-escola e primeira série utilizaram uma amostra composta de 90 alunos da rede estadual de ensino que apresentavam desenvolvimento linguístico típico, isto é com desenvolvimento considerado padrão para a idade. Destes, 40 alunos eram da pré-escola, com idade média de seis anos e cinco meses, e 50 eram da primeira série, com idade média de sete anos e dois meses.

A amostra selecionada foi submetida à avaliação das habilidades de memória de trabalho com base no Modelo de Memória de Trabalho de Baddeley (2000), envolvendo o componente fonológico. O componente fonológico foi avaliado através do subteste cinco, Memória Seqüencial Auditiva, do Teste Illinois de Habilidades Psicolinguísticas (ITPA), adaptação brasileira realizada por Bogossian e Santos (1977), e da Prova de Repetição de Palavras sem Significado, elaborado por Kessler (1997). (GENDRI et al, 2007, p. 313) 
As habilidades de consciência fonológica foram estudadas a partir do teste Consciência Fonológica: "Instrumento de Avaliação Sequencial (CONFIAS), elaborado por Moojen et al (2003), considerando tarefas de consciência silábica e fonêmica. A escrita foi caracterizada conforme a proposta de Ferreiro e Teberosky (1999)." (apud GENDRI 2007, p. 313).

Os resultados destas pesquisam indicam que os pré-escolares apresentaram capacidade de repetir sequências de dígitos e sílabas menor que os alunos de primeira série (nominalização anterior ao sistema de ensino de nove anos), mas considerado compatível com a idade; em consciência fonológica, o desempenho em nível de sílabas e em nível de fonemas também foi menor que os alunos de primeira série; o mesmo ocorreu em relação a hipótese de escrita, em sua maioria.

As pesquisadoras Gendri et al (2007) concluíram que o desempenho em memória de trabalho, consciência fonológica e nível de escrita se inter-relacionam, bem como estão relacionados com a idade cronológica, a maturidade e a escolaridade.

A memória operacional fonológica e o desenvolvimento de linguagem em crianças com desenvolvimento normal de linguagem foram estudados e pesquisados por Rodrigues e Lopes (2009) com o objetivo de descrever e discutir sobre a avaliação da memória operacional fonológica em crianças com desenvolvimento normal. As pesquisadoras realizaram uma revisão sistemática da literatura sobre memória operacional fonológica e sua relação com o desenvolvimento das habilidades de linguagem em crianças normais. Utilizando como fonte de pesquisa livros, monografias, teses, dissertações e artigos publicados nas bases de dados Lilacs, Pubmed, Scielo e Medline. A partir das pesquisas e leituras chegaram à conclusão que existe uma relação entre o conhecimento fonológico e lexical e a memória operacional fonológica em crianças em desenvolvimento normal de linguagem.

Em relação à gestão do conhecimento, Eckschmidt et al (2007) em um trabalho intitulado "Memória de Trabalho e Inteligências Múltiplas na Construção da Gestão do Conhecimento" procuraram mostrar qual o papel da memória de trabalho e a sua relação com as inteligências múltiplas em contextos propícios para a gestão do conhecimento. Discutindo o conceito geral da memória em uma perspectiva histórica, biológica e neurofisiológica concluem que há relações intrínsecas entre aspectos de memória de trabalho com a gestão do conhecimento.

A gestão do conhecimento também envolve habilidades cognitivas que favoreçam o desenvolvimento da linguagem e aquisição das habilidades de leitura e 
escrita. Vaz et al (2010) ao pesquisarem memória de trabalho em crianças utilizando a Tarefa de Brown-Peterson para realizar a avaliação das crianças, com o objetivo de verificar o desenvolvimento da memória de trabalho ao longo das séries iniciais do ensino fundamental e identificar se há adequação da Tarefa de Brown Peterson na avaliação desta função afirmam que:

O desempenho na Tarefa de Brown Peterson apresentou função crescente ao longo das séries e faixas etárias. $\mathrm{O}$ aumento no tempo de interferência produziu diminuição linear na pontuação em todas as séries. Correlações positivas foram encontradas entre o desempenho na Tarefa de Brown Peterson e Dígitos, sendo que Tarefa de Brown Peterson se mostrou mais sensível para diferenciar as séries. Conclusão: o estudo confirmou que a maturação da memória de trabalho continua ao longo do ensino fundamental, indicando maturação tardia das áreas cerebrais relacionadas. A Tarefa de Brown Peterson se mostrou um instrumento adequado para a avaliação de memória de trabalho em crianças. (VAZ et al, 2010, p.63).

Participaram do estudo 103 crianças, com a idade média de 9,75 anos, da primeira à sexta série do ensino fundamental. Entre os participantes, 63 eram do sexo masculino. As crianças foram avaliadas segundo a Tarefa de Brown Peterson, Dígitos Ordem Direta e Dígitos Ordem Inversa. O desempenho foi comparado em função das variáveis: sexo; idade e série escolar.

Estudo realizado por Lopes et al (2005) sobre memória de trabalho e capacidade viso-espacial em crianças de 7 a 12 anos mostrou que todos os fatores principais alcançaram significância estatística. As crianças mais velhas tiveram uma freqüência de acertos maior que as crianças mais novas. Os estímulos dos conjuntos com similaridade baixa foram mais bem recordados que os estímulos com similaridade alta. A taxa de recordação foi melhor nas provas em que as letras de um conjunto foram todas apresentadas com a mesma cor, assim como a porcentagem de respostas corretas variou de forma significativa em função da posição espacial dos estímulos. Setenta e oito crianças participaram do experimento em que foram manipulados os fatores idade, posição espacial, similaridade visual e cor dos estímulos memorizados.

\subsubsection{Resultados}

A partir da revisão de literatura feita constatamos que existem vários núcleos dedicados a pesquisa da memória, alguns se dedicam com mais especificidade a 
memória de trabalho e suas possíveis relações com os campos do conhecimento, prejuízos ou doenças diversas e áreas da linguagem.

Verificamos também que a grande maioria das pesquisas voltadas aos aspectos da memória, independente de ser relacionados a aspectos linguísticos ou não, não são realizadas por centros de pesquisas de linguagem ou departamentos de linguística, letras e afins. A grande maioria dos laboratórios de pesquisas está associada aos centros de Psicologia, Medicina e Fonoaudiologia.

A partir do recorte de tempo e dos critérios que adotamos, encontramos no Brasil pesquisas do laboratório de Psicologia da Universidade Federal de Minas Gerais, Belo Horizonte - FAFICH-UFMG, pesquisas referentes inteligência e memória de trabalho: a relação entre fator $\mathrm{g}$, complexidade cognitiva e capacidade de processamento, validação da bateria de avaliação da memória de trabalho, Colom e Mendoza (2006), (BAMT-UFMG) e do departamento de Clínica Médica, da mesma universidade, encontramos pesquisas sobre memória de trabalho em pacientes com doença de Parkinson: características clínicas Beato et. al(2008). Na Universidade de Uberlândia - UFU - identificamos um trabalho sobre memória de trabalho viso-espacial em crianças de 7 a 12 anos, Lopes et. al (2007).

As pesquisas em São Paulo concentram-se no Núcleo de Neurociência e Comportamento do Instituto de Psicologia da Universidade de São Paulo e Departamento de Psiquiatria da Faculdade de Medicina da Universidade de São Paulo associado ao do Hospital das Clínicas da Faculdade de Medicina da Universidade de São Paulo - USP e Ribeirão Preto; Primi (2002); Lukasova et. al (2009); Berberian et. al(2008); Messina e Tieadermann (2009). Estes núcleos de São Paulo parecem ser os que apresentam maior número de pesquisas em andamento e com maior número de resultados e pesquisas publicados. Desenvolvem pesquisas com crianças, jovens e adultos, tanto com desenvolvimento normal quanto com diversos distúrbios. Identificamos, por exemplo, trabalhos que tratam da discriminação fonológica e memória em crianças com dislexia e bons leitores; memória operacional fonológica e suas relações com o desenvolvimento da linguagem infantil; caracterização da linguagem e memória de trabalho em pacientes com síndrome epiléptica;

Ainda em São Paulo, na Universidade São Francisco - Itatiba - encontramos trabalho de pesquisa voltado para inteligência fluida: definição fatorial, cognitiva e neuropsicológica. 
$\mathrm{Na}$ universidade de Brasília encontramos estudos dos departamentos de Fonoaudiologia e de Psicologia sobre Memória de trabalho, Gendri et. al (2007) que analisa a consciência fonológica e hipótese de Escrita e sobre Memória de trabalho em crianças avaliada pela Tarefa de Brown-Peterson Vaz et. al(2009).

No Rio Grande do Sul, Figueiredo et. al (2007) do departamento de Psicologia da Universidade de Pelotas desenvolvem pesquisas e avaliações de memória a partir de testes psicológicos que avaliam a capacidade de desempenhar tarefas que envolvem a linguagem. Na Universidade Federal - URGS, pesquisadores dedicam-se na busca de elementos que comprovem a importância da memória de trabalho na gestão do conhecimento.

Em Santa Catarina, na Universidade Federal - UFSC, também são desenvolvidas pesquisas referentes à importância da memória de trabalho na gestão do conhecimento no departamento de Engenharia, Eckschmidt et. al (2006). O mesmo departamento também pesquisa a memória de trabalho e inteligências múltiplas na construção da gestão do conhecimento. Outros trabalhos relacionados à memória e linguagem são desenvolvidos no Centro Comunicação e Expressão no Programa de PósGraduação em Linguística.

Aspectos dedicados especificamente a questões de memória e linguagem desenvolvidos em centros de linguística encontramos na Universidade Católica de Pelotas (UCPel) que desenvolveu estudo que faz comparação entre o desempenho de crianças bilíngues e monolíngues em tarefas envolvendo a memória de trabalho. Na Universidade Federal de Santa Catarina - UFSC- existem estudos que procuram identificar a capacidade de memória de trabalho na produção e aquisição de uma regra sintática na fala em L2. No departamento de Linguística e Língua Portuguesa da PUCMG, identificamos pesquisas sobre os segredos da memória no cérebro: dos princípios fundamentais da atenção, emoção e recategorização às relações analógicas.

Em outros países contemplados pelo banco de dados da SciELO localizamos pesquisas desenvolvidas na Venezuela, na Escuela de Ingeniería de Sistemas, CEMISID, Universi dad de Los Andes e no Departamento de Computación, Universidad Simón Bolívar. Sartenejas-Barut.

No Chile, na Académica Pontificia Universidad Católica de Valparaíso localizamos uma pesquisa direcionada à Conciencia metapragmática y memória operativa en niños escolares. 
No México, Laboratório de Aminoácidos Excitadores, Instituto Nacional de Neurologia e Neurocirurgia Manuel Velasco Suarez localizamos trabalhos sobre sistemas de memória, suas classificações e conceitos; Sistemas de memória de longo prazo: Memória episódica, sistemas de memória não declarativa e memória de trabalho. outro estudo denominado: "Transducción de la señal, pivote de la integración neurobiológica de la memoria. Una propuesta diferente a la tradicional hipótesis del sistema colinérgico" da Unidad de Investigación Biomolecular del Hospital de Cardiología UIBCAR), Hospital de Cardiología del Centro Médico Nacional (CMN) Siglo XXI, Instituto Mexicano del Seguro Social, México, D.F., México

\section{CONSIDERAÇÕES FINAIS}

A relação entre memória e comportamento é muito complexa, frequentemente apresenta controversas entre os pesquisadores, contudo é aceita como certa, não há como negar que ela não exista. O nosso conhecimento sobre esta fundamental relação é ainda muito limitado, mas os principais contornos e muitos dos detalhes das correlações entre cérebro e comportamento já foram suficientemente bem explicadas para serem úteis no contexto clínico.

O que se percebe a partir dos estudos encontrados é que qualquer comportamento humano é produto de uma infinidade de complexas interações bioquímicas e neurofisiológicas que envolvem todo o cérebro. Estudos sobre estimulação elétrica têm demonstrado de forma precisa particularidades funcionais de certas áreas cerebrais, agrupamentos de células cerebrais e de algumas células nervosas isoladas, dando-nos uma visão inicial das peças funcionais do comportamento. É neste emaranhado que se constituem as nossas memórias.

Os dados encontrados na pesquisa apontam para grupos distintos de estudos voltados para diferentes aspectos da memória, principalmente da memória de trabalho e suas relações com diferentes aspectos comportamentais.

Os grupos de pesquisa voltados para a velocidade de processamento de aspectos da aprendizagem e fator de inteligência sugerem que todo o nosso conhecimento e as nossas habilidades são armazenados em diferentes formas de memória, permitindo a nossa orientação no tempo e no espaço sendo a memória de trabalho a responsável pela manutenção do controle e atenção.

Em relação ao outro grupo pesquisado, os que associam os aspectos de memória a algum prejuízo ou doença, como, por exemplo, Parkinson, Alzheimer, déficit de 
atenção, esquizofrenia, síndrome epiléptica, dislexia, apontam para problemas de memória, ou seja, sujeitos com baixa capacidade de manutenção da memória de trabalho demoram mais tempo para dar uma resposta e que as patologias acima citadas provocam perdas e sérios prejuízos na memória de trabalho.

Por fim, o grupo de trabalhos que pesquisa especificamente aspectos linguísticos, em centros de linguística, indicam que, por exemplo, a capacidade de memória de trabalho está relacionada com a produção de uma estrutura sintática na fala de uma segunda língua ou L2 e fortemente relacionada com o processo de aquisição da língua. Outro fator importante na linguagem como apontam as pesquisas, é que há uma relação entre o conhecimento fonológico e lexical e a memória de trabalho em crianças em desenvolvimento normal da linguagem.

Portanto, concluímos, desta forma, que a memória de trabalho não é apenas o armazenamento de informações, mas um processo contínuo de recategorização e reorganização por meio de recursos provenientes da própria condição de fluxo de energia dos seres vivos e dependendo do que cada indivíduo se propõem a fazer, ou do prejuízo que tenha sofrido, poderá apresentar diferentes variações para a memória e seus comportamentos.

\section{REFERÊNCIAS}

AGUILAR CASTRO, J. L. ; SUMOZA MATOS, R. L. Protocolo de coherencia de memoria cache para sistemas distribuidos. Rev. Téc. Ing. Univ. Zulia. [online]. vol.30, $\mathrm{n}^{\mathrm{o}} .2$, ago. 2007.

BERBERIAN, A. A. et. al Avaliação da memória de trabalho na esquizofrenia e sua correlação com habilidades de funções executivas. Ver. Bras. Psiquiatria, vol. 31, n. 3, p.219-26, Jun./2009.

CARNEIRO, Maria Paula. Desenvolvimento da memória na criança: o que muda com a idade? Psicol. Reflex. Crit. [online]. vol.21, n.1, p. 51-59, 2008. ISSN 0102-7972.

CARRILLO-MORA, P. Sistemas de memoria: reseña histórica, clasificación y conceptos actuales. Segunda parte: Sistemas de memoria de largo plazo: Memoria episódica, sistemas de memoria no declarativa y memoria de trabajo. Salud Ment [online]. vol.33, n.2, pp. 197-205, 2010. ISSN 0185-3325.

COLOM R., MENDOZA C. F. Armazenamento de Curto Prazo e Velocidade de Processamento Explicam a Relação entre Memória de Trabalho e o Fator $g$ de Inteligência. Psicologia: Teoria e Pesquisa. Vol. 22 n. 1, p. 113-122, Jan-Abr 2006. 
Inteligência e Memória de Trabalho: A Relação Entre Fator $G$, Complexidade Cognitiva e Capacidade de Processamento. Psicologia: Teoria e Pesquisa, Vol. 17 n. 1, p. 037-047, Jan-Abr 2001.

CRESPO ALLENDE, N. , ALVARADO BARRA, C. Conciencia metapragmática y memoria operativa en niños escolares. Lit. lingüíst. [online]. n.21, pp. 93-108, 2010,. ISSN 0716-5811.

ECKSCHMIDT, A. et. al Memória de Trabalho e Inteligências Múltiplas na Construção da Gestão do Conhecimento. 2007. Disponível em:

http://www.ssia.cefetmg.br/site/sobre/aux/edicoes_ant/SSIA_2.html. Acesso em: jul 2011.

FUKUDA M. T. H. et. al Characterization of language and phonological working memory in patients with myoclonic astatic epileptic syndrome. Arq Neuropsiquiatria. n. 68 v. 1, p. 30-34, 2010.

GINDRI, G.; KESKE-SOARES, M.; MOTA, H. B. Memória de trabalho, consciência fonológica e hipótese de escrita. Pró-Fono Revista de Atualização Científica, Barueri (SP), v. 19, n. 3, p. 313-322, jul.-set. 2007.

LOPES E.J. et al. Memória de trabalho viso-espacial em crianças de 7 a 12 anos. Estudos de Psicologia, n. 10, v. 2, p.207-214, 2005.

LUKASOVA K., BARBOSA A. C. C., MACEDO E. C. de. Discriminação fonológica e memória em crianças com dislexia e bons leitores. Psico-USF, v. 14, n. 1, p. 1-9, jan./abr. 2009.

MANSILLA-OLIVARES, A. Transducción de la señal, pivote de la integración neurobiológica de la memoria: Una propuesta diferente a la tradicional hipótesis del sistema colinérgico. Gac. Méd. Méx [online]. vol.141, n.6, p. 513-526, 2005,. ISSN 0016-3813.

MATLIN, M. W. Psicologia Cognitiva. 5. ed: LTC. Rio de Janeiro. 2004.

MESSINA, L. de F; TIEDEMANN, K. B. Avaliação da memória de trabalho em crianças com transtorno do déficit de atenção e hiperatividade. Psicologia USP, n.20, v.2, p.209-228, São Paulo, abril/junho, 2009.

MIRANDA et. al A importância da memória de trabalho na gestão do conhecimento. Ciência e Cognição, vol 09, p. 111-119, 2006. Disponível em:

WWW.cienciaecognicao.org.

PRIMI, R. Inteligência fluida: definição fatorial, cognitiva e neuropsicológica. Paidéia, n. 12, v.23, p.57-75, 2002.

RODRIGUES A, BEFI-LOPES DM. Memória operacional fonológica e suas relações com o desenvolvimento da linguagem infantil. Pró-Fono Revista de Atualização Cientifica. n. 21, v.1, p. 63-68, jan-mar/2009. 
SILVEIRA, R., FINARDI, K. Working memory capacity in the production and acquisition of a syntactic rule in L2 speech. RBLA, Belo Horizonte, v. 11, n. 1, p. 199221, 2011.

TESSMANN BANDEIRA, M. H. Comparação entre o desempenho de crianças bilíngües e monolíngües em tarefas envolvendo a memória de trabalho. Anais do CELSUL 2008. Disponível em: http://www.ufrgs.br/ppgletras/celsul/index.htm .

VASCONCELOS, M.; ALBUQUERQUE, P. B. Dissociações entre tarefas de memória: Evidência para uma distinção entre as memórias implícita e explícita. Aná. Psicológica. [online]. vol.24, no.4 [citado 16 Julho 2011], p.519-532, out. 2006,

VAZ, I. A. CORDEIRO, P.M.; MACEDO, E.C. L. K. Memória de trabalho em crianças avaliada pela Tarefa de Brown-Peterson. Pró-Fono Revista de Atualização Científica. n. 22, v. 2, p.95-100, abr-jun 2010.

WOOD, G. M. de O.; CARVALHO, M. R. S.; ROTHE-NEVES, R. and HAASE, V. G. Validação da Bateria de Avaliação da Memória de Trabalho (BAMT-UFMG). Psicol. Reflex. Crit. [online]. vol.14, n.2, p. 325-341. 2001. ISSN 0102-7972. 